\title{
The COMPASS results on longitudinal spin effects and future measurements
}

\author{
Marcin Stolarski ${ }^{\mathrm{a}}$, On behalf of COMPASS collaboration \\ ${ }^{a}$ LIP-Lisboa, Av. Elias Garcia 14, 1 1000-149 Lisboa, Portugal
}

\begin{abstract}
A short review of the COMPASS results obtained in inelastic polarized muon scattering off the longitudinally polarized proton and deuteron targets is given. The measured gluon polarization inside the nucleon is presented including the final value of $\Delta G / G$ from the open charm LO analysis. New results on $A_{1}^{p}$ and the Bjorken Sum Rule test are shown. Finally the plans and goals of the future COMPASS measurements are presented.
\end{abstract}

Keywords:

Delta G/G, gluon polarization, nucleon structure, Bjorken sum rule

\section{Introduction}

COMPASS is an experiment at CERN focusing on the spin structure of the nucleon and hadron spectroscopy. Between 2002 and 2004, a $160 \mathrm{GeV} / \mathrm{c}$ polarized muon beam and a two cell polarized ${ }^{6} \mathrm{LiD}$ target were used for spin studies. For the 2006 data taking major upgrades of the spectrometer were made. This included of the RICH improvement for particle ID, a new COMPASS solenoid around the target with an angular acceptance increased from $70 \mathrm{mrad}$ to $180 \mathrm{mrad}$, and a new three cell target. In 2006 the ${ }^{6} \mathrm{LiD}$ target was used as it is optimal for $\Delta G / G$ measurement. In 2007 an ammonia $\mathrm{NH}_{3}$ target was used so that spin effects on polarized protons could be studied.

COMPASS has a rich physics program. Two more COMPASS talks were given in this conference: transverse spin effects were presented by C. Schill and for hadron spectroscopy please refer to work by J. Bernhard. In this paper selected results for longitudinal spin effects will be presented. This includes measurement of the gluon polarization inside the nucleon, spin dependent structure functions, quark helicity distribution and finally ideas for further COMPASS measurements.

\section{Gluon polarization}

The EMC results [1] in the late 80s suggested that the quarks may be responsible for only a small fraction of

Email address: mstolars@cern.ch (On behalf of COMPASS collaboration)

Preprint submitted to Nuc. Phys. (Proc. Suppl.) the nucleon spin contrary to the naive expectation that the spin of the nucleon may be built from valence quarks only. Several other experiments in world leading laboratories of particles physics (at CERN, DESY, JLAB and SLAC) confirmed the EMC observation with improved precision. The contribution of quarks to the nucleon spin is now believed to be about $30 \%$. More generally the spin of the nucleon can be decomposed as:

$$
S_{z}=\frac{1}{2}=\frac{1}{2} \Delta \Sigma+\Delta G+L_{q}+L_{g},
$$

where $\Delta \Sigma$ and $\Delta G$ are the polarized quark and gluon contributions to the nucleon spin and $L_{q, g}$ are their angular momenta. In COMPASS so far the gluon polarization is measured by studying photon-gluon fusion processes (PGF). This can be done in $i$ ) open charm studies and ii) in the analysis of hadrons produced with high transverse momenta (HipT). In the former case the analysis is free from any physical background. It is believed that the charm quarks are not constituents of the nucleon and so the appearance of the charm mesons in the final state suggest that a PGF process took place. Unfortunately due to the large charm mass, in the COMPASS energy range the production cross section for charm mesons is small also branching ratio for the $D^{0} \rightarrow K \pi$ is below $4 \%$. Therefore the analysis has limited statistical precision. The latter method has an advantage over open charm that the production cross-section is larger therefore the statistical error of $\Delta G / G$ will be reduced. On the other hand the HipT analysis is not background free. Even at the highest $p_{T} s$ there are contributions from leading order and QCD Compton processes. In

September 27, 2010 
addition for low $Q^{2}$ events the resolved photon contribution has to be included, which may reach $50 \%$ of the cross section. Almost all the crucial ingredients for this analysis have to be obtained from MC simulation using generators like LEPTO or PYTHIA. Therefore in this analysis the systematic error plays a very important role. In this paper the older results will be only mentioned while the new open charm analysis will be discussed in detail.

In the current open charm analysis all available data from 2002-2007 were used. In order to improve the statistical error of $\Delta G / G$ five different $D$ mesons decay modes are studied. In total there are about $65000 D^{0}$ candidates and about $29000 D^{*}$ candidates out of which 13000 are in the golden channel $D^{*} \rightarrow K \pi \pi_{\text {slow }}$.

In a simplified approach the gluon polarization $\Delta G / G$ can be obtained from:

$$
\frac{\Delta G}{G}=\frac{1}{P_{T} P_{b} f a_{L L} \frac{S}{S+B}} A^{\mu N \rightarrow D^{0}+X}
$$

where $P_{T, b}$ are the target and beam polarization, respectively, $f$ is the dilution factor of the material which takes into account the fraction of polarizable nucleons in the target, including radiative corrections. $a_{L L}$ is the so called analysing power which represents the polarization transfer from muon to photon and from gluon to charm quarks. The $\frac{S}{S+B}$ is the ratio between signal and signal plus combinatorial background, and finally the $A^{\mu N \rightarrow D^{0}+X}$ is the measured asymmetry. The method which is actually used in the analysis is much more complex e.g. it allows the simultaneous extraction of signal and background asymmetries, details can be found in [2]. The typical spectra for all five discussed channels are shown in the Fig. 1.

To increase the statistical significance of the results the events are weighted on an event by event basis. The weight for each event is $\frac{1}{P_{T} P_{b} f a_{L L}} \frac{S}{S+B}$. In the current analysis $S /(S+B)$ is parametrized using a Neural Network approach. As a model of background the wrong charge combinations (wcc) are used e.g. $K^{+} \pi^{+}$, while the $D^{0}$ decays into $K^{+} \pi^{-}$a good charge combination ( $g c c$ ). The NN is trying to find the differences between $w c c$ and $g c c$ in the phase space of the input parameters which can be attributed to the $D^{0}$ mesons present in the good charge combination sample. The input parameters for $\mathrm{NN}$ are e.g. $E_{D^{0}}, z_{D^{0}}$, particle PID quality. An example of NN parametrization is shown in Fig. 2. As a function of $S /(S+B)$ as obtained from NN the $D^{*}$ spectrum is plotted. As one observes the higher $S /(S+B)$ from NN the larger the observed peak in real physics data.
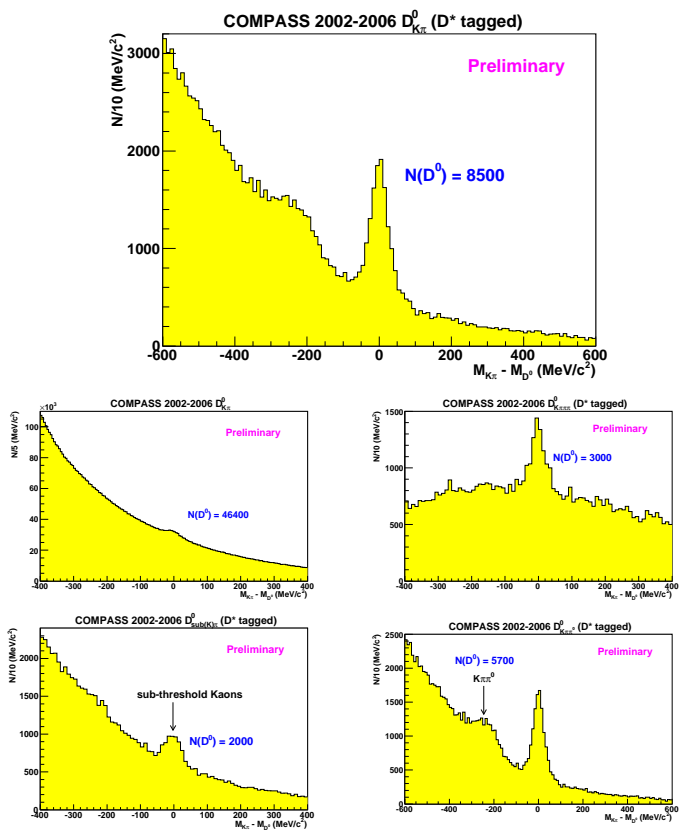

Figure 1: The mass spectra for $D^{0}$ mesons in five analyzed channels. Data from 2002-2006 are presented.

The final result $\Delta G / G=-0.08 \pm 0.21 \pm 0.11$ is compatible with zero and the error bar is reduced by a factor of 1.3 w.r.t. the previously published value [2]. The current value is about 0.4 higher than previously published. With the additional years and new channels included in the analysis, and taking into account the correlation between the two analyses, the resulting change corresponds to a bit more than two sigma. We treat it as a statistical fluctuation. The results from five channels are summarized in Table 1.

Table 1: The $\Delta G / G$ results from COMPASS open charm analysis.

$\begin{array}{ll}\text { channel } & \Delta G / G \\ D^{*} \rightarrow K \pi \pi_{\text {slow }} & -0.19 \pm 0.30 \\ D^{0} \rightarrow K \pi & +0.02 \pm 0.42 \\ D^{*} \rightarrow K \pi \pi^{0} \pi_{\text {slow }} & -0.41 \pm 0.58 \\ D^{*} \rightarrow K 3 \pi \pi_{\text {slow }} & +0.63 \pm 0.83 \\ D^{*} \rightarrow K_{\text {subth }} \pi \pi_{\text {slow }} & +0.5 \pm 1.0 \\ \text { TOTAL } & -0.08 \pm 0.21(\text { stat. }) \pm 0.11(\text { sys. })\end{array}$

The COMPASS results on $\Delta G / G$ from HipT analysis are the following. The preliminary result for $Q^{2}>1$ $(\mathrm{GeV} / \mathrm{c})^{2} 2002-2004$ is $\Delta G / G=0.08 \pm 0.10 \pm 0.05$. The hard scale of the process is ensured by $Q^{2}>1(\mathrm{GeV} / \mathrm{c})^{2}$ cut, therefore one can be less strict about hadrons transverse momenta cuts. The analysis is still in progress. The improved method of analysis allows an error reduction by about $30 \%$. In addition the 2006 data are being 


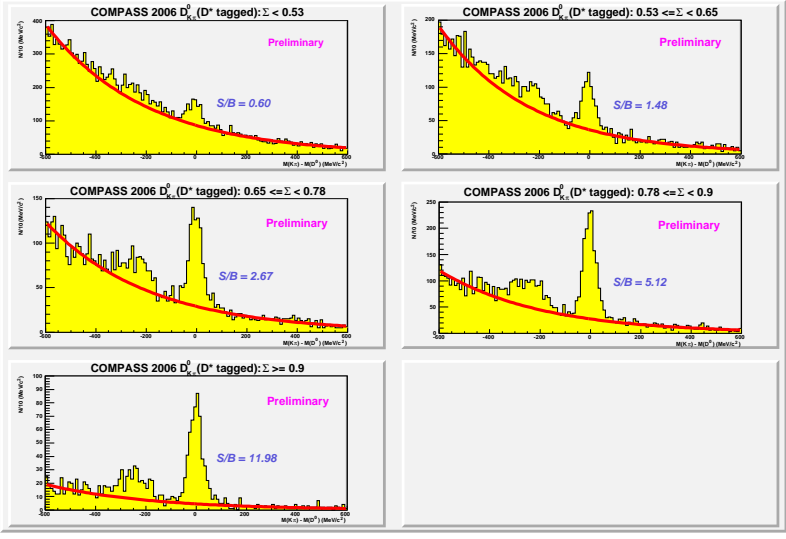

Figure 2: Example of $\mathrm{S} /(\mathrm{B}+\mathrm{S})$ parametrization by $\mathrm{NN}$ for $D^{*}$ channel

analyzed.

The preliminary result for $Q^{2}<1(\mathrm{GeV} / \mathrm{c})^{2} 2002$ 2004 is $\Delta G / G=0.016 \pm 0.058 \pm 0.014 \pm 0.052 \pm 0.013$, where the errors account for statistical, systematic related to spectrometer, $\mathrm{MC}$ and from resolved photon contribution respectively. The analysis in the low $Q^{2}$ region requires strict cuts on transverse momenta of the hadrons to ensure that perturbative QCD can be used. The results of the two analyses are in good agreement.

The summary of the $\Delta G / G$ measurements is presented in Fig. 3. The results suggest that the gluon contribution to the nucleon spin is rather small. The plotted curves are the QCD NLO fit done by COMPASS [3].

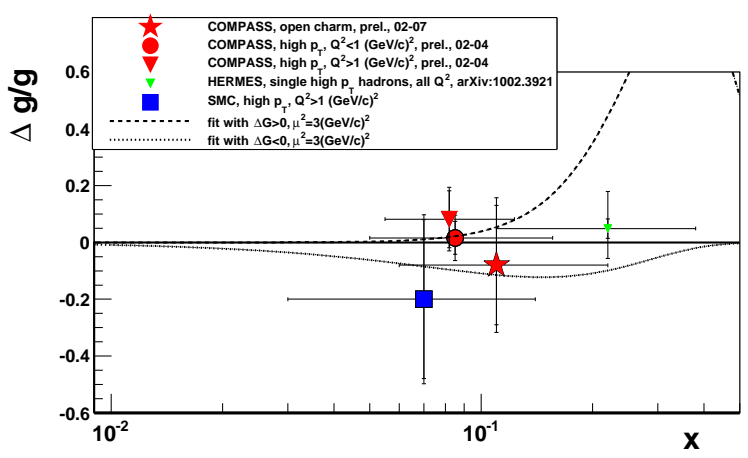

Figure 3: Summary of measurements of $\Delta G / G\left(x_{G}\right)$.

\section{COMPASS inclusive measurements}

As mentioned in the introduction in 2007 COMPASS for the first time measured the spin effects on the proton target $\left(\mathrm{NH}_{3}\right)$. One of the first results was the measurement of the spin dependent asymmetry $A_{1}^{p}$ and the spin dependent structure function $g_{1}^{p}$. The results for $A_{1}^{p}$ are presented in Fig. 4. They are in good agreement with other experiments. It is worth to notice that in the low $x$ region, with only one year of data taking, the statistical errors are better than those from any other previous measurement.

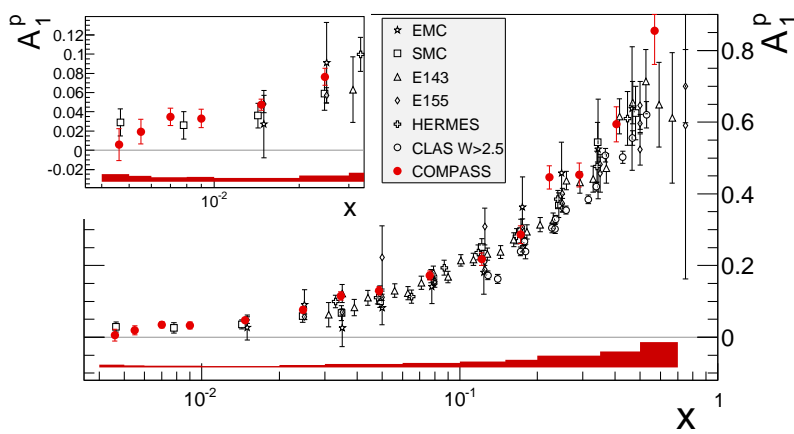

Figure 4: Comparison of COMPASS $A_{1}^{p}$ with world data for $\mathrm{Q}^{2}>1$ $(\mathrm{GeV} / \mathrm{c})^{2}$

Having measured both $g_{1}^{p}$ and $g_{1}^{d}$ COMPASS could study the so called non-singlet spin structure function $g_{1}^{N S}\left(x, Q^{2}\right)=g_{1}^{p}\left(x, Q^{2}\right)-g_{1}^{d}\left(x, Q^{2}\right)$. This function is of special interest because its $Q^{2}$ evolution does not depend upon very poorly known gluon spin densities. According to the fundamental Bjorken sum rule the integral of $g_{1}^{N S}\left(x, Q^{2}\right)$ is proportional to the ratio of $g_{A} / g_{V}$ the axial and vector coupling constants.

$$
\int_{0}^{1} g_{1}^{N S}\left(x, Q^{2}\right)=\Gamma_{1}^{N S}=\frac{1}{6} \frac{g_{A}}{g_{V}} C_{1}^{N S}\left(Q^{2}\right)
$$

where $C_{1}^{N S}\left(Q^{2}\right)$ has been calculated in pQCD up to $\alpha_{s}^{3}\left(Q^{2}\right)$. The $\left|g_{A} / g_{V}\right|$ was precisely measured in the $\beta$ neutron decay and the value is $\left|g_{A} / g_{V}\right|=1.2694 \pm 0.0028$ [4]. In Fig. 5 the integral of $\int_{x}^{1} g_{1}^{N S}\left(x, Q^{2}\right) d x$ is shown. In the limit of the available phase space in $x_{B j}$ the result is in agreement with the expected value. The COMPASS result is $\left|g_{A} / g_{V}\right|=1.28 \pm 0.07$ (stat. $) \pm 0.10$ (syst. [5] in very good agreement with the above, but with much larger error bars.

COMPASS is also analysing the semi-inclusive asymmetries for kaons and pions on both proton and deuteron targets. As a result of the analysis one study flavour separated the quark helicity distributions. Some results were already published in [6]. One of the most interesting helicity distributions is the one of strange quarks $\Delta s(x)$. It is known from inclusive measurements to be negative. On the other hand in the semi-inclusive analysis the measured values in various experiments are very close to zero without a negative tendency. In the 


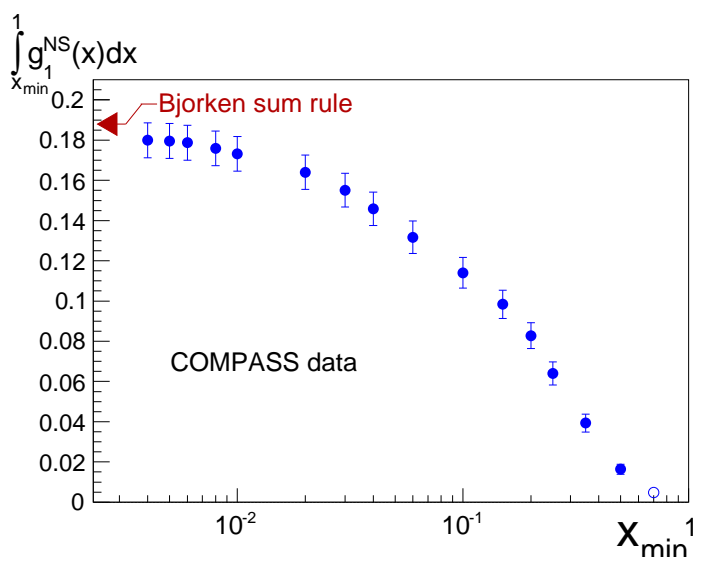

Figure 5: Bjorken sum rule test from COMPASS data.

cited paper it was shown that $\Delta s$ and its error strongly depends upon the choice of fragmentation functions. At the moment COMPASS is trying to extract some ratios of fragmentation functions so that one of the uncertainties in the $\Delta s$ measurement will be removed.

\section{COMPASS further plans.}

The COMPASS collaboration has submitted a new proposal to CERN scientific committees [7]. The main goals of the proposed experiment, are the transverse momentum dependent structure functions (TMDs) measurement in Drell-Yan process and generalized parton distribution functions (GPDs) measurement via the DVCS process. The analysis of these processes will improve our knowledge about the structure of the nucleon. Certain physical observables accesible in these processes are related with the angular momentum of the quarks inside nucleon, therefore the $L_{q}$ therm in the Eq. 1 can be studied.

In the last years COMPASS performed short test runs to prove the feasibility of the proposed measurements. As an example in the results from the Deep Virtual Compton Scattering (DVCS) test are shown. The studied process is therefore exclusive photon production in $\mu p \rightarrow \mu p \gamma$. This process has a natural background from the so called Bethe-Heitler process (radiative effects). The process is fully calculable from theory. In Fig. 6 the angle between $\gamma$ and the lepton scattering plane is shown for different $x_{B j}$ ranges. The shape of the $\mathrm{BH}$ process can be calculated analytically and is rather peaked around the zero angle, while the DVCS process has a flat distribution. The obtained results suggest that the DVCS events are observed in COMPASS.
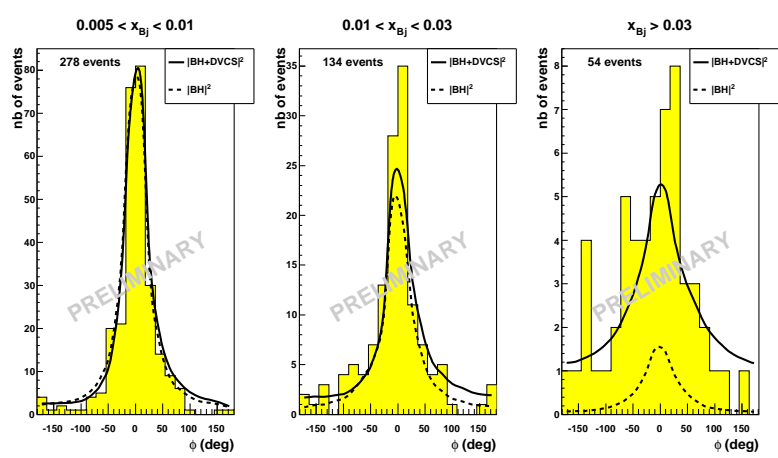

Figure 6: 2009 test run; The measured angle between $\gamma$ and the lepton scattering plane in $\mu p \rightarrow \mu p \gamma$.

\section{Summary}

The updated COMPASS result on $\Delta G / G$ from open charm was presented. The final value is $\Delta G / G=$ $-0.08 \pm 0.21$ (stat.) \pm 0.11 (sys.). The Bjorken sum rule was tested in COMPASS, the resulting $\left|g_{A} / g_{V}\right|$ is in agreement with results of more precise experiments. Finally after successful test runs COMPASS submitted a new proposal to CERN.

\section{Acknowledgments}

This research was partially supported by the Portuguese Fundacao para a Ciencia e a Tecnologia (FCT).

\section{References}

[1] EMC, J. Ashman et al., Nucl. Phys. B 328 (1989) 1; Phys. Lett. B 206 (1988) 364.

[2] COMPASS, M. Alekseev et al., Phys. Lett. B 676, (2009) 31

[3] COMPASS, V.Yu. Alexakhin et al., Phys. Lett. B 647, (2007) 330

[4] C.Amsler et al. (Particle Data Group), Phys. Lett. B 667 (2008) 1.

[5] COMPASS, M.G. Alekseev et al., Phys. Lett. B 690 (2010) 466.

[6] hep-ex/1007.4061 and COMPASS, M. Alekseev et al., Phys. Lett. B 680 (2009) 216,

[7] http://wwwcompass.cern.ch/compass/proposal/compassII_proposal/compass-II_proposal.pdf 